Pacific Journal of Mathematics

ON STRATIFYING PAIRS OF LINEAR MAPPING

Vol. 102, No. 2

February 1982 


\title{
ON STRATIFYING PAIRS OF LINEAR MAPPINGS
}

\author{
C. G. GIBSON AND T. D. WARD
}

The complex linear representations (of fixed dimension) of an oriented graph form a finite dimensional vector space $M$ with a natural action of a product $G$ of general linear groups. It is interesting to look for natural Whitney stratifications of $M$ invariant under $G$. For the Dynkin diagrams $A_{n}, D_{n}, E_{6}, E_{7}, E_{8}$ such stratifications are provided by the orbits; and for the extended Dynkin diagrams $\widetilde{A}_{n}, \widetilde{D}_{n}, \tilde{E}_{6}$, $\tilde{E}_{7}, \tilde{E}_{8}$ one might expect to obtain such stratifications by 'neglecting moduli', in an obvious way. This is known to be the case for $\widetilde{A}_{0}$. For $\widetilde{A}_{1}$ we show that this procedure does yield a stratification, and that at least the regular strata satisfy the Whitney conditions.

A question of general interest in singularity theory is whether, given a Lie group $G$ acting differentiably on a differentiable manifold $M$ (we are thinking primarily of the algebraic case) there exists a natural stratification of $M$, invariant under the action, subject at least to the proviso that all the orbits in a given stratum have the same dimension. A more difficult question is whether there exist Whiney stratifications [8] with the same properties.

An interesting class of examples where these questions are decidedly nontrivial arises in the study of complex linear representations of finite graphs. The general situation is as follows. One starts with a finite connected graph $\Gamma$, allowing the possibility that two vertices may be joined by several edges, or that an edge may join a vertex to itself. It is assumed that $\Gamma$ is endowed with a fixed orientation, i.e., to each edge $e$ is assigned a vertex $\alpha(e)$ called the starting point, and a vertex $\beta(e)$ called the end point: moreover it is assumed that the orientation is admissible, in the sense that there do not exist circuits in the graph with the end point of each edge the starting point of the next edge. Now suppose that to each vertex $\alpha$ of $\Gamma$ is assigned a finite dimensional complex vector space $U_{\alpha}$, yielding a family $U=\left(U_{\alpha}\right)$, and that to each edge $e$ is assigned a linear mapping $f_{e}: U_{\alpha(e)} \rightarrow U_{\beta(e)}$, yielding a family $f=\left(f_{e}\right)$. One refers to the pair $(U, f)$ as a complex linear representation of the oriented graph $\Gamma$. One can take the complex linear representations to be the objects of a category $\mathscr{L}(\Gamma)$ : a morphism $\phi:(U, f) \rightarrow$ $(V, g)$ is then a family of linear mappings $\phi: U_{\alpha} \rightarrow V_{\alpha}$ with the property that for any edge $e$ the following diagram of linear mappings commutes 


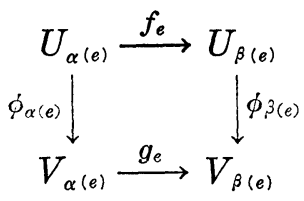

One can define the direct sum of two objects $(U, f)$ and $(V, g)$ to be the object $(W, h)$ where $W_{\alpha}=U_{\alpha} \oplus V_{\alpha}$ and $h_{e}=f_{e} \oplus g_{e}$. A nonzero object $(U, f)$ is then indecomposable when it cannot be written as the direct sum of two nonzero objects. By the Krull-RemakSchmidt theorem [4] any object $(U, f)$ in the category $\mathscr{L}(\Gamma)$ is isomorphic to the direct sum of finitely many indecomposable objects, unique up to order and isomorphism.

The situation of particular interest to us is the following. Suppose that to each vertex $\alpha$ is assigned a nonnegative integer $n_{\alpha}$, and that we restrict our attention to those objects for which $V_{\alpha}=$ $\boldsymbol{C}^{n_{\alpha}}$. These objects then form a finite dimensional complex vector space $M$ on which one has a natural differentiable action of a Lie group $G$, namely a product of general linear groups $G L\left(n_{\alpha}, C\right)$ each corresponding to changes of basis in $V_{\alpha}$ : and the isomorphism types in the category correspond to the orbits under this action. Note that the action is algebraic so that by well-known results [3] the orbits are constructible submanifolds of $M$. We pose now the general question of the opening paragraph for this class of examples, and look for restrictions which will at least provide us with candidates for natural stratifications.

The obvious approach here is to follow the classification of graphs in the algebraic theory. In the early 1970's it was shown that there are only finitely many indecomposable objects (up to isomorphism) in the category $\mathscr{L}(\Gamma)$ if and only if $\Gamma$ is one of the Dynkin diagrams $A_{n}(n \geqq 1), D_{n}(n \geqq 4), E_{\theta}, E_{7}$ or $E_{8}$ : a proof of this result, and a description of the indecomposable objects was given in [2]. Thus for the Dynkin diagrams there are only finitely many orbits in $M$, providing the required stratification: indeed, since the orbits are constructible one has a Whitney stratification [8], hence a complete answer to the original question.

The next stage in the algebraic theory is to consider the extended Dynkin diagrams $\widetilde{A}_{n}(n \geqq 0), \widetilde{D}_{n}(n \geqq 4), \widetilde{E}_{6}, \widetilde{E}_{7}$ and $\widetilde{E}_{8}$. Here one identifies two types of indecomposable, namely discrete and homogeneous. The discrete indecomposables are classified up to isomorphism, but the homogeneous indecomposables only up to dimension. An account of the extended Dynkin diagrams can be found in [5] and [6]. Now we ask for a candidate for a natural stratification of $M$ for the extended Dynkin diagrams. This time we cannot simply take the orbits as the strata, as they are no 
longer locally finite, and have to look more closely. For each homogeneous indecomposable one has a "modulus" appearing, and the obvious way of producing a finite partition of $M$ invariant under the action is just to neglect moduli. We conjecture that this provides a stratification of $M$ for all the extended Dynkin diagrams, and probably a Whitney stratification. The simplest example here is the extended Dynkin diagram $A_{0}$ illustrated in Fig. 1 . Here $M$ can be thought of as the space of all complex $n \times n$ matrices, $G$ as

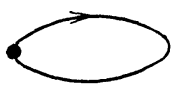

Figure 1

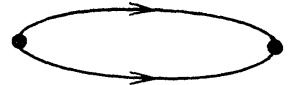

FIgURE 2

the group of all invertible complex $n \times n$ matrices, and the action $G \times M \rightarrow M$ is given by $(P, A) \rightarrow P^{-1} A P$ with the orbits equivalence classes of $n \times n$ matrices, under the relation of similarity. In this example only homogeneous indecomposables occur, namely Jordan matrices, and the partition of $M$ obtained by neglecting moduli is precisely the Segre stratification of [7]: there it was shown that the partition is indeed a Whitney stratification of $M$, so establishing our conjecture for the extended Dynkin diagram $\widetilde{A}_{0}$. The next simplest example is $\widetilde{A}_{1}$, illustrated in Fig. 2. In this case $M$ is the space of all pairs of linear mappings $\boldsymbol{C}^{m} \rightarrow \boldsymbol{C}^{n}$, under the action of change of basis in domain and target. The problem of finding the indecomposables was solved by Kronecker in 1890; he obtained four types, two discrete and two homogeneous, and it is the interaction of these types which renders $\widetilde{A}_{1}$ a significantly harder example than $\tilde{A}_{0}$ from our viewpoint. In this paper we shall prove that the partition of $M$ obtained by neglecting moduli is indeed a stratification of $M$. We are unable as yet to prove that this is a Whiteny stratification, though we do show that the so-called regular strata form a Whitney stratification. In another paper [11] we manage to get some way towards understanding the nonregular strata by producing a complete list of specializations between strata: see [10].

1. The Kronecker stratification. For practical purposes it is convenient to work not with the linear mappings themselves, but rather with their matrices relative to the standard bases. Thus we take $M=M(m, n)$ to be the complex vector space of all pairs $(A, B)$ of complex $m \times n$ matrices. And we take the Lie group $G$ to be the product of the complex general linear groups $G L(m) \times$ $G L(n)$. The action $\Phi: G \times M \rightarrow M$ is then given by

$$
\Phi((P, Q),(A, B))=(P A Q, P B Q) .
$$


The next step is to describe the canonical indecomposables in the category of linear representations of the extended Dynkin diagram $\widetilde{A}_{1}$. For this it will be convenient to introduce some special matrices. We set

$$
P_{k}=\left(\begin{array}{c}
I_{k} \\
0
\end{array}\right)_{k+1, k} \quad Q_{k}=\left(\begin{array}{c}
0 \\
I_{k}
\end{array}\right)_{k+1, k}
$$

with $I_{k}$ the identity $k \times k$ matrix: and for any complex number $\lambda$ we set

$$
J(k ; \lambda)=\left(\begin{array}{llll}
\lambda & & & \\
1 & \lambda & & \\
& \cdot & \cdot & \\
& & & 1
\end{array}\right)_{k \times k}
$$

With this notation it turns out that any indecomposable is isomorphic to one of the following four types [6]

$$
\begin{array}{lll}
\text { type I: } & \hat{k}=\left(P_{k}, Q_{k}\right) & (k \geqq 0) \\
\text { type II: } & k=\left(P_{k}^{T}, Q_{k}^{T}\right) & (k \geqq 0) \\
\text { type III: } & k^{*}(\mu)=\left(J(k, \mu), I_{k}\right) & (k \geqq 1) \\
\text { type IV: } & k(\lambda)=\left(I_{k}, J(k, \lambda)\right) & (k \geqq 1) .
\end{array}
$$

Note that when $\mu=0$ a canonical indecomposable of type III with modulus $\mu$ is isomorphic to one of type IV with modulus $\mu^{-1}$ : these provide the only isomorphisms in the above list. Types I, II are discrete whilst types III, IV are homogeneous, in the sense of [5]. Any matrix pair which is a direct sum of canonical indecomposables will be called a canonical element: thus any matrix pair is isomorphic to some canonical element, whose summands are unique, up to order. It will be convenient to reserve the term regular element for an element of $M$ for which the canonical element to which it is isomorphic contains only indecomposables of types III, IV: the reason for this is that the regular elements form an open subset of $M$, in the Zariski topology, for which the partition we are about to introduce can actually be proved to be a Whitney stratification.

To describe the partition of $M$ we require more notation. First, for a sequence $a=\left(a_{1}, \cdots, a_{s}\right)$ of positive integers with $a_{1} \leqq a_{2} \leqq \cdots$ $\leqq a_{s}$, and a complex number $\lambda$, we introduce the block diagonal Jordan matrix

$$
J(a ; \lambda)=\operatorname{diag}\left(J\left(a_{1}: \lambda\right), \cdots, J\left(a_{s}: \lambda\right)\right) .
$$

Suppose now that we are given nondecreasing sequences of nonnegative integers $\sigma_{1}=\left(p_{1}, \cdots, p_{a}\right), \sigma_{2}=\left(q_{1}, \cdots, q_{b}\right)$, sets of non- 
decreasing sequences of positive integers $\sigma_{3}=\left\{r_{1}, \cdots, r_{c}\right\}, \sigma_{4}=$ $\left\{s_{1}, \cdots, s_{d}\right\}$ distinct complex numbers $\mu_{1}, \cdots, \mu_{c}$, and distinct complex numbers $\lambda_{1}, \cdots, \lambda_{d}$ subject to the proviso that we have $\lambda_{i} \mu_{j} \neq 1$ for all indices $i, j$. To these we associate the canonical elements

$$
E \sigma_{1}=\bigoplus_{i=1}^{n} \hat{p}_{i}: E \sigma_{2}=\bigoplus_{i=1}^{h} q_{i}
$$

and

$$
\begin{aligned}
& E \sigma_{3}\left(\mu_{1}, \cdots, \mu_{c}\right)=\bigoplus_{i=1}^{\dot{\theta}}\left(J\left(r_{i} ; \mu_{i}\right), I\right) \\
& E \sigma_{4}\left(\lambda_{1}, \cdots, \lambda_{d}\right)=\bigoplus_{i=1}^{f}\left(I, J\left(s_{i} ; \lambda_{i}\right)\right)
\end{aligned}
$$

where in all cases the identity matrix $I$ has the same order as the Jordan block matrix. Now any matrix pair is certainly isomorphic to a canonical element of the form

$$
E \sigma_{1} \oplus E \sigma_{2} \oplus E \sigma_{3}\left(\mu_{1}, \cdots, \mu_{c}\right) \oplus E \sigma_{4}\left(\lambda_{1} \cdots, \lambda_{d}\right)
$$

Moreover, if two such canonical elements are isomorphic they must have the same $\sigma_{1}, \sigma_{2}$, the same $c+d$, and the same $\sigma_{5}=\sigma_{3} \cup \sigma_{4}$. One refers to the $p_{i}, \cdots, p_{a}$ as the minimal row indices, and the $q_{1}, \cdots, q_{b}$ as the minimal column indices: $\sigma_{1}$ and $\sigma_{2}$ are the minimal row and column index types. Finally we dub $\sigma_{5}$ the invariant factor type, and take the triple $\sigma=\left(\sigma_{1}, \sigma_{2}, \sigma_{5}\right)$ to be the canonical type.

The set $\sum_{\sigma}$ of all matrix pairs in $M$ of a given canonical type will be called a stratum in $M$ : this yields a finite partition of $M$, which we shall dub the Kronecker stratification, and our main objective is to prove that this partition is indeed a stratification of $M$. Note particularly that with these definitions the canonical indecomposables

$$
\left(J(k ; 0), I_{k}\right) \quad\left(I_{k}, J(k, \lambda)\right)
$$

always belong to the same stratum. Note also that the strata are constructible subsets of $M$ : this uses a standard argument based on Chevalley's theorem, and is written out in full in [10].

In practice one needs to be able to compute the canonical type of a given matrix pair $(A, B)$. A procedure for calculating the minimal indices was first described in [12], and goes as follows. Introduce sequences of matrices $M_{1}, \cdots, M_{m}$ and $N_{1}, \cdots, N_{n}$ by taking 


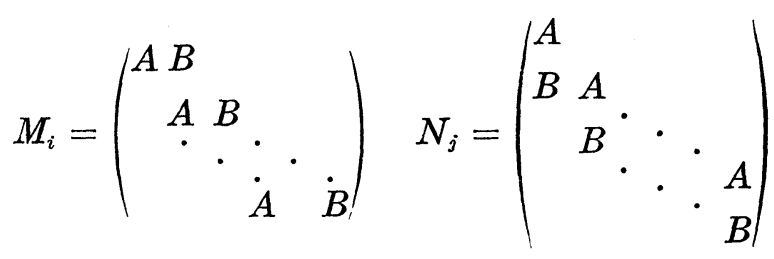

where $A, B$ appear $i$ times in $M_{i}$, and $j$ times in $N_{j}$. Now define sequences of integers $\alpha_{1}, \cdots, \alpha_{m}$ and $\beta_{1}, \cdots, \beta_{n}$ by setting

$$
\left\{\begin{array}{l}
\alpha_{i}=i m-\operatorname{rank}\left(M_{i}\right) \\
\beta_{j}=j n-\operatorname{rank}\left(N_{j}\right) .
\end{array}\right.
$$

It then turns out that the number of minimal row indices of value $k$ is exactly $\alpha_{k+1}+\alpha_{k-1}-2 \alpha_{k}$, and that the number of minimal column indices of value $k$ is exactly $\beta_{k+1}+\beta_{k-1}-2 \beta_{k}$.

The computation of the invariant factor type of a matrix pair $(A, B)$ is a more familiar process. To the pair one associates the matrix $X A+Y B$ over the ring of homogeneous complex polynomials in $X, Y$. Write $D_{k}(X, Y)$ for the greatest common divisor of all $k \times k$ minors of $X A+Y B$ and set

$$
E_{k}(X, Y)=\frac{D_{k}(X, Y)}{D_{k-1}(X, Y)}
$$

for $1 \leqq k \leqq r$, where $r$ denotes the rank of $X A+Y B$. The homogeneous polynomials $E_{1}(X, Y), \cdots, E_{r}(X, Y)$ are the invariant factors of the matrix pair $(A, B)$ : each can be factorized as a product of powers of distinct linear factors, and these powers are the elementary divisors. Now suppose that the elementary divisors divisible by $Y$ are $Y^{r_{i}}$ with $1 \leqq i \leqq c$, and set $\sigma_{3}=\left\{\left(r_{1}, \cdots, r_{c}\right)\right\}$. And suppose that the remaining elementary divisors are $\left(X+\lambda_{i} Y\right)^{s_{i}, j}$ with $1 \leqq i \leqq d$, $1 \leqq j \leqq v_{i}$, say: set $\sigma_{4}=\left\{s_{1}, \cdots, s_{d}\right\}$ where $s_{i}=\left(s_{i, 1}, \cdots, s_{i, v_{i}}\right)$. It now follows from [6] that the matrix pair $(A, B)$ is isomorphic to the canonical element

$$
E_{1} \oplus E_{2} \oplus E_{3}(0) \oplus E_{4}\left(\lambda_{1}, \cdots, \lambda_{d}\right)
$$

with $\sigma_{1}$ and $\sigma_{2}$ the minimal row and column index types.

2. Construction of versal unfoldings. In subsequent sections it will be necessary for us to have explicit versal unfoldings [9] of elements of $M$ under the action of $G$. Thus we need to know the tangent space to the orbit through an element $(A, B)$, and an explicit supplement for the tangent space in $M$.

(2.1) The tangent space at $(A, B)$ to its orbit is the subspace 
of $M$ comprising all pairs ( $P A+A Q, P B+B Q$ ) with $P, Q$ arbitrary square matrices of orders $m, n$.

Proof. The natural mapping $G \rightarrow M$ onto the orbit given by $(P, Q) \rightarrow(P A Q, P B Q)$ is a submersion [9]: thus the required tangent space is the image of the differential of this map, at the identity in $G$, which is the mapping $(P, Q) \rightarrow(P A+A Q, P B+B Q)$ with $P, Q$ arbitrary square matrices of orders $m, n$.

Now we have to construct a supplement to the tangent space (of complementary dimension). A simple device to achieve this end is to introduce a scalar product on $M$, and compute the normal space. We define the scalar product of two elements $(A, B)$ and $(C, D)$ in $M$ to be

$$
\langle(A, B),(C, D)\rangle=\operatorname{tr}\left(A C^{*}\right)+\operatorname{tr}\left(B D^{*}\right)
$$

where $\operatorname{tr}$ denotes the trace, and * the adjoint matrix.

(2.2) The normal space at $(A, B)$ to its orbit is the subspace of $M$ comprising all pairs $(U, V)$ which satisfy the equations

$$
U A^{*}+V B^{*}=0: A^{*} U+B^{*} V=0 \text {. }
$$

Proof. $(U, V)$ lies in the normal space if and only if it is orthogonal to every vector in the tangent space, i.e., for all square matrices $P, Q$ of orders $m, n$ we have

$$
\langle(U, V),(P A+A Q, P B+B Q)\rangle=0 .
$$

A line or two of manipulation shows that this is equivalent to

$$
\operatorname{tr}\left(\left(U A^{*}+V B^{*}\right) P^{*}\right)+\operatorname{tr}\left(\bar{Q}\left(A^{*} U+B^{*} V\right)^{T}\right)=0
$$

for all square matrices $P, Q$ of orders, $m, n$. The result follows.

The general situation now is that one has a canonical element

$$
(A, B)=\left(A_{1}, B_{1}\right) \oplus \cdots \oplus\left(A_{p}, B_{p}\right)
$$

with $\left(A_{i}, B_{i}\right)$ a canonical indecomposable in $M\left(m_{i}, n_{i}\right)$ and one requires an explicit description of the matrix pairs $(U, V)$ in the normal space to the orbit at $(A, B)$. Suppose that the $(i, j)$ th block entry of $(U, V)$ is $\left(U_{i j}, V_{i j}\right)$ in $M\left(m_{i}, n_{j}\right)$. It is then a trivial consequence of (2.2) that we require

$$
U_{i j} A_{j}^{*}+V_{i j} B_{j}^{*}=0: A_{i}^{*} U_{i j}+B_{i}^{*} V_{i j}=0
$$

for all indices $i, j$. One now has to compute $U_{i j}, V_{i j}$ explicitly for each possible pair $\left(A_{i}, B_{i}\right)$ and $\left(A_{j}, B_{j}\right)$ of canonical indecomposables. 
Since there are four types of canonical indecomposable we have sixteen cases to consider. In every case the above homogeneous system of linear equations (in the entries of $U_{i j}$ and $V_{i j}$ ) solves rather easily: in some cases the solution space is trivial, but in all other cases one can pick out a list of entries which form a basis for the solution space. Now let $S_{i j}, T_{i j}$ denote the matrices obtained from $U_{i j}, V_{i j}$ by setting equal to zero all the entries, except for the basis entries: and let $(S, T)$ be the matrix pair whose $(i, j)$ th block entry is $\left(S_{i j}, T_{i j}\right)$. Notice that the scalar product of $(U, V)$ and $(S, T)$ is nonzero, so that the subspace of matrices $(S, T)$ has trivial intersection with the tangent space at $(A, B)$ to its orbit: but the subspace of matrix pairs $(S, T)$ has the same dimension as the subspace of matrix pairs $(U, V)$ so forms a supplement to the tangent space. The advantage of the matrix pairs $(S, T)$ over the $(U, V)$ is that they have far fewer nonzero entries, an important practical consideration in view of the sheer complexity of some of the versal unfolding we shall have to consider. The results of the computations are presented in the following table: we omit the computations themselves, which were written out in full in [10], as they are perfectly elementary, and consume undue space. Each entry in the columns headed $S_{i j}, T_{i j}$ is a condition on two positive integers $a, b$ and means that only the $(a, b)$ th entries satisfying this condition are allowed to be nonzero: there are no further conditions on the entries. When an entry involves only one of $a, b$ it is tacitly understood that the other can take all possible values. A zero entry 0 indicates that the matrix in question is the zero matrix.

\begin{tabular}{|c|c|c|c|c|}
\hline$\left(A_{i}, B_{2}\right)$ & $\left(A_{j}, B_{j}\right) \mid$ & $S_{i j}$ & $T_{\imath j}$ & conditions \\
\hline \multirow[b]{2}{*}{$\hat{p}_{1}$} & \multirow{2}{*}{$\hat{p}_{2}$} & 0 & 0 & $p_{2}-p_{1}-1 \leqq 0$ \\
\hline & & 0 & $\begin{array}{c}a=1 \\
1 \leqq b \leqq p_{2}-p_{1}-1\end{array}$ & $p_{2}-p_{1}-1>0$ \\
\hline$\hat{p}$ & $q$ & $a=p+1 \quad \& \quad b=1$ & $a=1$ or $b=1$ & none \\
\hline$\hat{p}$ & $r^{*}(\mu)$ & $a=q+1$ & 0 & none \\
\hline$\hat{p}$ & $s(\lambda)$ & 0 & $a=1$ & none \\
\hline$q$ & $\hat{p}$ & 0 & 0 & none \\
\hline \multirow[b]{2}{*}{$q_{1}$} & \multirow[b]{2}{*}{$q_{2}$} & 0 & 0 & $q_{1}-q_{2}-1 \leqq 0$ \\
\hline & & $\begin{array}{c}1 \leqq a \leqq q_{1}-q_{2}-1 \\
b=1\end{array}$ & 0 & $q_{1}-q_{2}-1>0$ \\
\hline$q$ & $r^{*}(\mu)$ & 0 & 0 & none \\
\hline
\end{tabular}




\begin{tabular}{|c|c|c|c|c|}
\hline$\left(A_{i}, B_{i}\right)$ & $\left(A_{j}, B_{j}\right)$ & $S_{i j}$ & $T_{j i}$ & conditions \\
\hline$q$ & $s(\lambda)$ & 0 & 0 & none \\
\hline$r^{*}(\mu)$ & $\hat{p}$ & 0 & 0 & none \\
\hline$r^{*}(\mu)$ & $q$ & $b=1$ & 0 & none \\
\hline \multirow{3}{*}{$r_{1}^{*}(\mu)$} & \multirow{3}{*}{$r_{2}^{*}(\mu)$} & 0 & 0 & $\mu_{1} \neq \mu_{2}$ \\
\hline & & $b=r_{2}$ & 0 & $\begin{array}{l}\mu_{1}=\mu_{2} \\
r_{1} \leqq r_{2}\end{array}$ \\
\hline & & $a=1$ & 0 & $\begin{array}{l}\mu_{1}=\mu_{2} \\
r_{1} \geqq r_{2}\end{array}$ \\
\hline$r^{*}(\mu)$ & $s(\lambda)$ & 0 & 0 & $\lambda \mu \neq 1$ \\
\hline$s(\lambda)$ & $\hat{p}$ & 0 & 0 & none \\
\hline$s(\lambda)$ & $q$ & 0 & $b=q+1$ & none \\
\hline$s(\lambda)$ & $r^{*}(\mu)$ & 0 & 0 & none \\
\hline \multirow{3}{*}{$s_{1}\left(\lambda_{1}\right)$} & \multirow{3}{*}{$s_{2}\left(\lambda_{2}\right)$} & 0 & 0 & $\lambda_{1} \neq \lambda_{2}$ \\
\hline & & 0 & $b=s_{2}$ & $\begin{array}{l}\lambda_{1}=\lambda_{2} \\
s_{1} \leqq s_{2}\end{array}$ \\
\hline & & 0 & $a=1$ & $\begin{array}{l}\lambda_{1}=\lambda_{2} \\
s_{1} \geqq s_{2}\end{array}$ \\
\hline
\end{tabular}

Given $m, n$ and a canonical element $(A, B)$ it is now a purely mechanical matter to write down a versal unfolding of $(A, B)$ in the space $M(m, n)$. For instance when $m=3, n=2$ a versal unfolding of the canonical element $\hat{0}+2^{*}(\lambda)$ comprises all pairs of matrices

$$
\left(\begin{array}{ll}
0 & 0 \\
1 & 0 \\
0 & 1
\end{array}\right)\left(\begin{array}{cc}
s & t \\
\lambda+v & u \\
1 & \lambda+v
\end{array}\right)
$$

with sufficiently small unfolding parameters $s, t, u, v$. (This is not quite the unfolding obtained from the above table, but equivalent to it.) For fixed $v$ the unfolding space is 3 -dimensional with coordinates $s, t, u$. The unfolding has type $\hat{2}$ exactly on the complement of the cubic surface given by $t^{2}=u s^{2}$ : this is the so-called Whitney umbrella comprising a surface with two connected components, and a line, appropriately dubbed the handle. On the umbrella, but off the handle, the unfolding has type $\hat{1}+1^{*}(\lambda)$ : on the handle, but away from the origin, one obtains $\hat{1} \oplus 1^{*}(\lambda) \oplus 1^{*}(\lambda)$ : and finally at the origin one obtains precisely the original stratum. 


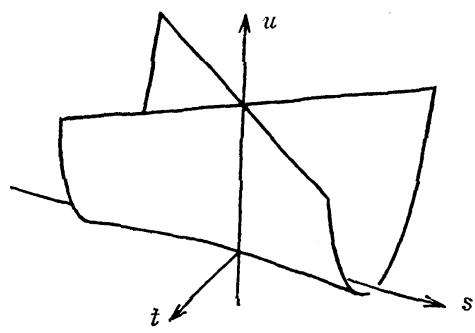

Figure 3

Of course one can systematically compute the codimensions of orbits by counting the number of unfolding parameters in the versal unfoldings constructed according to the above prescription. Unlike the case of $\widetilde{A}_{0}$ the codimension is not additive, in the obvious sense of the word, so when computing the codimension of a direct sum one has to take into account the unfolding terms which arise from the interaction of pairs of summands. A simple example of this is provided by the canonical element $\hat{p}_{1} \oplus \cdots \hat{p}_{a}$ with $p_{1} \leqq \cdots \leqq p_{a}$ : each $p_{i}$ has codimension zero, in the space of pairs of matrices of the same size, but the direct sum has codimension. $\sum_{i<j} p_{i j}$ where we write $p_{i j}=\max \left(0, p_{j}-p_{i}-1\right)$. And similarly the codimension of the canonical element $q_{1} \oplus \cdots \oplus q_{b}$ with $q_{1} \leqq \cdots \leqq q_{b}$ is $\sum_{i<j} q_{i j}$ with $q_{i j}=\max \left(0, p_{j}-p_{i}-1\right)$. Finally, the codimension of the mixed direct sum

$$
\hat{p}_{1} \oplus \cdots \oplus \hat{x}_{a} \oplus \underline{q}_{1} \oplus \cdots \oplus \underline{q}_{b}
$$

will be

$$
\sum_{i<j} p_{i j}+\sum_{i<j} q_{i j}+\sum_{i<j}\left(p_{i}+q_{j}+2\right) \text {. }
$$

3. The regular strata. In $\S 1$ we defined a regular element of $M(n, n)$ to be an element for which the canonical representative on the orbit is a direct sum of indecomposables of types III and IV. We shall say that a stratum $\sum$ in $M(n, n)$ is regular when one, and hence every, element contained in it is regular. Note that an element $(A, B)$ is regular if and only if the matrix $X A+Y B$ has nonzero determinant. Thus the regular elements in $M(n, n)$ form an open set; moreover, only regular strata can specialize to a regular stratum, i.e., contain it in their frontiers. What we intend to prove is

(3.1) The Kronecker stratification of the regular elements in $M(n, n)$ is a Whitney stratification.

Let us concentrate first on showing that any regular stratum 
$\sum$ is indeed a submanifold of $M(n, n)$. The proof is based on a well-known lemma [7] that it suffices to show that $\sum$ appears as a manifold in a versal unfolding of any element $(A, B)$. Indeed it will turn out that $\sum$ appears as an affine subspace in the unfolding. Moreover, the method of proof will also yield Whitney regularity. Our starting point is

(3.2) Let $(A, B)$ be a direct sum of canonical indecomposables of type III, all having the same eigenvalue $\mu$; and let $(P, Q)$ be any matrix-pair in the versal unfolding of $(A, B)$ constructed in $\S 2$. Then the highest common factor $\Delta_{k}(X, Y)$ of all the $k \times k$ minors of $X P+Y Q$ is a homogeneous polynomial of degree $k$ in $X, \mu X+Y$ whose coefficients are all products of unfolding parameters, and hence independent of the eigenvalue $\mu$.

Proof. By the construction of $\S 2$ the matrix-pair $(P, Q)$ is, in a natural way, a block matrix-pair $\left(P_{i j}, Q_{i j}\right)$. And the construction yields exactly three possibilites for the form of the matrix $X P_{i j}+$ $Y Q_{i j}$, depicted below. The symbol * simply denotes an unfolding parameter.

$$
\begin{aligned}
& \left(\begin{array}{ccc}
\mu X+Y+{ }^{*} X & { }^{*} X \cdots \cdots \cdots *^{*} X \\
\vdots & \mu X+Y \cdots \cdots \cdot 0 \\
\vdots & \vdots & \vdots \\
0 & 0 \cdots \cdots \cdots \cdot+Y
\end{array}\right) \\
& \text { Case } i=j
\end{aligned}
$$

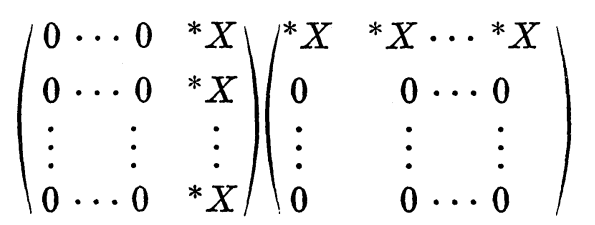

$$
\begin{aligned}
& \text { Case } i<j \quad \text { Case } i>j
\end{aligned}
$$

The proposition now follows immediately by sheer inspection of the matrices.

(3.3) Let $\left(A^{\prime}, B^{\prime}\right)$ be a direct sum of canonical indecomposables of type IV, all having the same eigenvalue $\lambda$; and let $\left(P^{\prime}, Q^{\prime}\right)$ be any matrix-pair in the versal unfolding of $\left(A^{\prime}, B^{\prime}\right)$ constructed in $\S 2$. Then the highest common factor $\Delta_{k}^{\prime}(X, Y)$ of all the $k \times k$ minors of $X P^{\prime}+Y Q^{\prime}$ is a homogeneous polynomial of degree $k$ in $X+\lambda Y, Y$ whose coefficients are products of unfolding parameters, and hence independent of $\lambda$. 
The proof of (3.3) follows exactly the same lines as that of (3.2). Consider now a general regular canonical element $(A, B)$. This we can certainly write in the form

$$
(A, B)=\bigoplus_{i=1}^{\llcorner}\left(A_{i}, B_{i}\right) \bigoplus_{j=1}^{d}\left(A_{j}^{\prime}, B_{j}^{\prime}\right)
$$

where each $\left(A_{i}, B_{i}\right)$ is of the type described in (3.2) with associated eigenvalue $\mu_{i}$, and each $\left(A_{j}^{\prime}, B_{j}^{\prime}\right)$ is of type described in (3.3) with associated eigenvalue $\lambda_{j}$. Moreover, we can assume that $\mu_{1}, \cdots, \mu_{c}$ are distinct, that $\lambda_{1}, \cdots, \lambda_{d}$ are distinct, and that $\mu_{i} \lambda_{j} \neq 1$ for all indices $i, j$. Observe now that the versal unfolding of $(A, B)$ constructed in $\S 2$ comprises matrix-pairs

$$
(P, Q)=\bigoplus_{i=1}^{c}\left(P_{i}, Q_{i}\right) \bigoplus_{j=1}^{d}\left(P_{j}^{\prime}, Q_{j}^{\prime}\right)
$$

with $\left(P_{i}, Q_{i}\right)$ in the versal unfolding of $\left(A_{i}, B_{i}\right)$, and with $\left(P_{j}^{\prime}, Q_{j}^{\prime}\right)$ in the versal unfolding of $\left(A_{j}^{\prime}, B_{j}^{\prime}\right)$. Now $(P, Q)$ must itself be regular, vide the opening remarks of this section, so by $\S 1$ its type depends only on the invariant factor type of $X P+Y Q$, i.e., the elementary divisors of its determinant. Let us write $D_{k}(X, Y), E_{k}^{(i)}(X, Y)$, $F_{k}^{(j)}(X, Y)$ respectively for the highest common factors of the $k \times k$ minors of $X P+Y Q, X P_{i}+Y Q_{i}, X P_{j}^{\prime}+Y Q_{j}^{\prime}$. Further, we write $s_{i}$ for the common order of the matrices $A_{i}, B_{i}$ and $t_{j}$ for the common order of the matrices $A_{j}^{\prime}, B_{j}^{\prime}$. By inspection we see that the determinants

$$
\begin{aligned}
& E_{s_{i}}^{(i)}(X, Y)=\left(\mu_{i} X+Y\right)^{s_{i}}+e_{i}\left(X, \mu_{i} X+Y\right) \\
& F_{t_{j}}^{(j)}(X, Y)=\left(X+\lambda_{j} Y\right)^{t_{i}}+f_{j}\left(X+\lambda_{j} Y, Y\right)
\end{aligned}
$$

where $e_{i}, f_{j}$ are homogeneous polynomials of degrees $s_{i}, t_{j}$, whose coefficients are products of unfolding parameters, so can be supposed as small as we please. It follows that we can suppose that no two of the $E_{s_{i}}^{(i)}$ have a common factor, since they certainly do not when all the unfolding parameters are zero: likewise we can suppose that no two of the $F_{t_{j}}^{(j)}$ have a common factor. Finally, it is clear that $E_{s_{i}}^{(1)}, F_{t_{j}}^{(i)}$ have no common factor. We use these facts as follows. For $0 \leqq k<n$ we have

$$
D_{n-k}(X, Y)=\prod_{i=1}^{c} E_{s_{i}-k}^{(i)}(X, Y) \prod_{j=1}^{d} F_{t_{i}-k}^{(j)}(X, Y)
$$

so that in view of (3.2), (3.3) and the remarks above the invariant factor type of $X P+Y Q$ is independent of the choices of eigenvalues $\mu_{1}, \cdots, \mu_{c}, \lambda_{1}, \cdots, \lambda_{d}$. Thus we have proved.

(3.4) Let $(A, B)$ be a regular canonical element: then the type 
of a matrix-pair $(P, Q)$ in its versal unfolding is invariant under small changes in the eigenvalues $\mu_{1}, \cdots, \mu_{c}, \lambda_{1}, \cdots, \lambda_{d}$.

The first consequence of this fact is that the stratum $\Sigma$ containing $(A, B)$ must be a manifold. We have already remarked in $\S 1$ that $\Sigma$ is certainly a constructible subset of $M(n, n)$. Thus the singular set of $\Sigma$ is of strictly lower dimension than $\sum$. But the homogeneity property expressed by (3.4) ensures that if $\sum$ has a singular point then the intersection of a neighborhood of that point with $\sum$ lies wholly in the singular set of $\sum$, yet has the same dimension as $\sum$. That contradiction establishes that $\sum$ is a nonsingular constructible set, hence a manifold. In fact this style of reasoning also establishes the Whitney regularity conditions over $\sum$. For this we have only to observe that by Whitney's theorem [8] the subset of $\sum$ where other strata fail to be Whitney regular over $\Sigma$ is constructible of strictly lower dimension, and that the homogeneity property expressed by (3.4) will certainly preserve Whitney regularity. That concludes the proof of (3.1).

It is well worth remarking that (3.1) can be extended to cover elements in $M(n+1, n)$ which are isomorphic to the direct sum of a single canonical indecomposable of type $I$ and a regular element; or dually to elements in $M(n, n+1)$ which are isomorphic to the direct sum of a single canonical indecomposable of type II and a regular element. The proof follows that of (3.1) very closely, and is written out in full in [10].

The computation of codimensions for regular strata in $M(n, n)$ is straightforward. Suppose we are given a finite list of distinct complex numbers $\alpha, \beta, \cdots$ and corresponding nondecreasing sequences of positive integers $a=\left(a_{1}, a_{2}, \cdots\right), b=\left(b_{1}, b_{2}, \cdots\right), \cdots$ giving rise to the regular element

$$
a_{1}(\alpha) \oplus a_{2}(\alpha) \oplus \cdots b_{1}(\beta) \oplus b_{2}(\beta) \oplus \cdots .
$$

Counting the number of unfolding parameters in the versal unfolding constructed in $\S 2$ we see that the codimension of the orbit containing the above normal form is precisely

$$
\left(a_{1}+3 a_{2}+5 a_{3}+\cdots\right)+\left(b_{1}+3 b_{2}+5 b_{3}+\cdots\right)+\cdots .
$$

And the codimension of the stratum containing the normal form will be obtained by deleting from this integer the number of distinct eigenvalues $\alpha, \beta, \cdots$, i.e., it will be

$$
\left(a_{1}+3 a_{2}+\cdots-1\right)+\left(b_{1}+3 b_{2}+\cdots-1\right)+\cdots .
$$

On the basis it is an easy matter to list the regular strata of 
low codimension in $M(n, n)$. Let us denote the stratum containing the normal form * by

$$
\alpha^{n_{1}} \alpha^{a_{2}} \cdots \beta^{b_{1}} \beta^{b_{2}} \ldots
$$

where $\alpha, \beta, \cdots$ are now regarded as dummy symbols. Note that the codimension of the stratum does not depend on the simple eigenvalues in the list $\alpha, \beta, \cdots$ i.e., those which appear with multiplicity 1; we can therefore adopt the convention of deleting from the above symbol those parts corresponding to simple eigenvalues. With this convention the strata of positive codimension $\leqq 4$ are given by the following table.

One can gain a very clear idea of how the strata of codimension $\leqq 3$ fit together by sketching their appearance in versal unfold-

\section{Codimension 1}

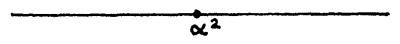

Codimension 2
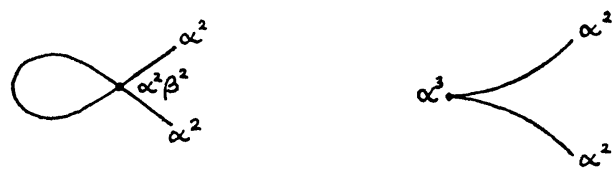

Codimension 3
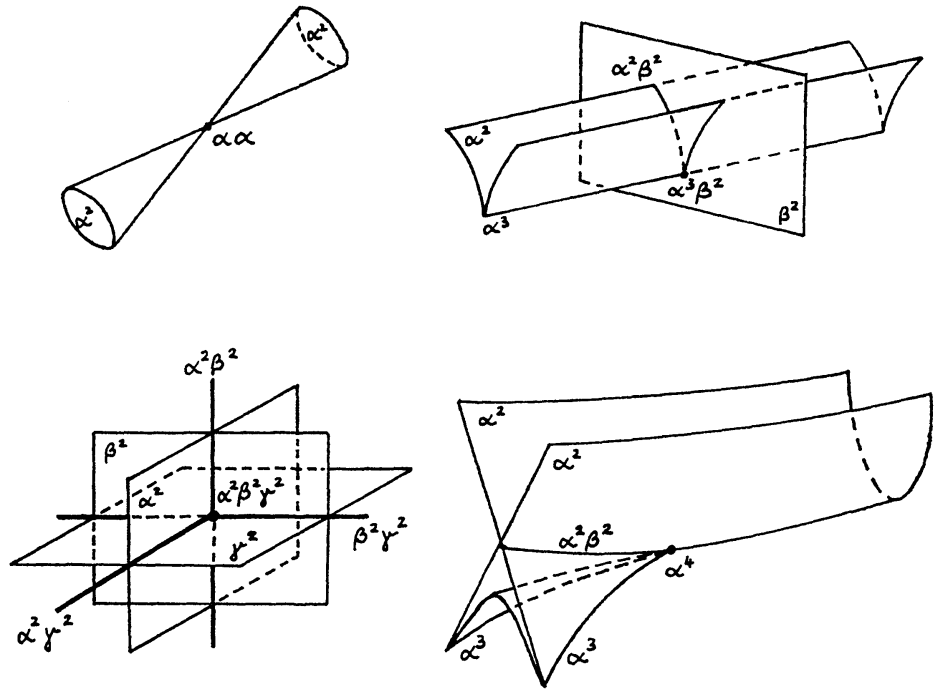

FIGURE 4 


\begin{tabular}{c|l}
\hline codimension & \multicolumn{1}{|c}{ strata having that cndimension } \\
\hline 1 & $\alpha^{2}$ \\
\hline 2 & $\alpha^{3}: \alpha^{2} \beta^{2}$ \\
\hline 3 & $\alpha^{4}: \alpha \alpha: \alpha^{3} \beta^{2}: \alpha^{2} \beta^{2} \gamma^{2}$ \\
\hline 4 & $\alpha^{5}: \alpha^{2} \alpha: \alpha^{4} \beta^{2}: \alpha \alpha \beta^{2}: \alpha^{3} \beta^{2} \gamma^{2}: \alpha^{3} \beta^{3}: \alpha^{2} \beta^{2} \gamma^{2} \delta^{2}$ \\
\hline
\end{tabular}

ings of normal forms-vide the example in $\S 2$. A certain amount of elementary computation gives rise to the following pictures, some of which are familiar from catastrophe theory, and were first obtained for the Segre stratification in [1].

4. Smoothness of the general stratum. It remains for us to prove that the general stratum $\Sigma$ in $M(n, m)$ is smooth, i.e., a differentiable (embedded) submanifold. As we observed above, it suffices to show that $\Sigma$ appears as a manifold in a versal unfolding of each element in $\Sigma$. We adhere to the notation of $\S 1$ and write $\Sigma_{o}$ for the stratum in $M(n, m)$ with canonical type $\sigma=\left(\sigma_{1}, \sigma_{2}, \sigma_{5}\right)$ where $\sigma_{5}=\sigma_{3} \cup \sigma_{4}$ : thus $\sum_{\sigma}$ contains the canonical element $E \sigma_{1} \oplus$ $E \sigma_{2} \oplus E \sigma_{3}(\mu) \oplus E \sigma_{4}(\lambda) \quad$ where $\quad \mu=\left(\mu_{1}, \cdots, \mu_{c}\right)$ and $\lambda=\left(\lambda_{1} \cdots, \lambda_{d}\right)$. Evidently, it suffices to show that the appearance of $\Sigma_{o}$ in a versal unfolding of this canonical element is diffeomorphic to the appearance of $\sum_{\left(\phi, \phi, \phi_{5}\right)}$ in a versal unfolding of $E \sigma_{3}(\mu)+E \sigma_{4}(\lambda)$, which is a manifold by the results of the previous section.

(4.1) Suppose $\sum$ has canonical type $\sigma$, and that $(P, Q)$ is an element of the versal unfolding of $E \sigma_{1} \oplus E \sigma_{2} \oplus E \sigma_{3}(\mu) \oplus E \sigma_{4}(\lambda)$ such that $(P, Q)$ also has canonical type $\sigma$. Then $(P, Q)=E \sigma_{1} \oplus E \sigma_{2} \oplus$ $\left(P^{\prime}, Q^{\prime}\right)$ where $\left(P^{\prime}, Q^{\prime}\right)$ has canonical type $\left(\phi, \phi, \sigma_{5}\right)$ and $i s$ an element of the versal unfolding of $E \sigma_{3}(\mu)+E \sigma_{4}(\lambda)$.

Proof. We sketch the main steps in the proof, suppressing the detail as it involves writing out rather complicated matrix pairs: a complete version of the proof appears in [10]. By examination of the table in $\S 2$ we see that the matrix $X P+Y Q$ has the form of a block matrix

$$
\left(\begin{array}{ccc}
U_{1} & U_{6} & U_{4} \\
0 & U_{2} & 0 \\
0 & U_{5} & U_{3}
\end{array}\right)
$$

where $U_{1}$ corresponds to $E \sigma_{1}, U_{2}$ corresponds to $E \sigma_{2}$, and $U_{3}$ corresponds to $E \sigma_{3}(\mu) \oplus E \sigma_{4}(\lambda)$.

The first step is to establish that $(P, Q)$ has canonical type $\sigma$ 
only if $U_{4}$ and $U_{5}$ are zero matrices. Suppose $\sigma_{1}=\left(p_{1}, \cdots, p_{a}\right), \sigma_{2}=$ $\left(q_{1}, \cdots, q_{b}\right)$ where $\sum p_{i}=p, \sum q_{j}=q$ and that $E \sigma_{3}(\mu) \oplus E \sigma_{4}(\lambda)$ is a $k \times k$ matrix. Then by examining the minors in $X P+Y Q$ of order $p+q+k$ one can check that the binary form $D_{p+q+k}(X, Y)$ has degree $k$ only if $U_{4}=0, U_{5}=0$ as was required. These minors are written out explicitly in [10].

The second step is to show that $U_{6}=0, U_{1}=E \sigma_{1}$ and $U_{2}=E \sigma_{2}$. Note that the stratum which contains $E \sigma_{1} \oplus E \sigma_{2}$ is a single orbit, and so the appearance of this stratum in the unfolding of $E \sigma_{1} \oplus E \sigma_{2}$ is the single point given by the vanishing of the unfolding parameters. Now

$$
\left(\begin{array}{cc}
U_{1} & U_{6} \\
0 & U_{2}
\end{array}\right)
$$

correponds to a matrix-pair in the unfolding of $E \sigma_{1} \oplus E \sigma_{2}$, by the observation immediately following (2.2), and so has canonical type $\left(\sigma_{1}, \sigma_{2}, \phi\right)$ only if $U_{1}=E \sigma_{1}, U_{2}=E \sigma_{2}$ and $U_{6}=0$. Further, $U_{3}$ corresponds to a regular matrix-pair, which does not therefore have any minimal row or column indices, so that $(P, Q)$ has canonical type $\sigma$ only if $U_{1}=E \sigma_{1}, U_{2}=E \sigma_{2}$ and $U_{6}=0$.

Finally, by the observation immediately following (2.2), $U_{3}$ corresponds to a matrix-pair $\left(P^{\prime}, Q^{\prime}\right)$, say, in the unfolding of $E \sigma_{3}(\mu) \oplus$ $E \sigma_{4}(\lambda)$. That completes the proof of (4.1), and hence the proof that the general stratum is smooth.

\section{REFERENCES}

1. V. I. Arnol'd, On matrices depending on parameters, Uspechi Mat. Nauk, 26 (1971), 101-114 (Russian Math. Surveys, 26 (1971), 29-43).

2. I. N. Bernstein, I. N. Gelfand, and B. A. Ponomarev, Coxeter functors and Gabriel's theorem, Uspechi Mat. Nauk 28 (1973), 19-33: translated in Russian Math. Surveys, 28 (1973), 17-32.

3. A. Borel, Linear Algebraic Groups, W. A. Benjamin Inc., New York, 1969.

4. C. W. Curtis and I. Reiner, Representation Theory of Finite Groups and Associative Algebras, Interscience, New York-London, 1962.

5. V. Dlab and C. M. Ringel, Representations of Graphs and Algebras, Carleton Math. Lecture Notes No. 8, 1974.

6. P. Gabriel, Representations indecomposables, Seminaire Bourbaki, 444 (1973/74).

7. C. G. Gibson, Regularity of the Segre stratification, Math. Proc. Cam. Phil. Soc., 80 (1976).

8. C. G. Gibson, K. Wirthmüller, A. A. du Plessis, and E. J. N. Looijenga, Topological Stability of Smooth Mappings, Springer Lecture Notes in Mathematics, 552, 1974.

9. C. G. Gibson, Singular Points of Smooth Mappings, Research Notes in Mathematics, Pitman, 1979.

10. T. D. Ward, On stratifying spaces of matrix pairs, Thesis, University of Liverpool, 1979 .

11. T. D. Ward and C. G. Gibson, Specializations for the Kronecker stratification, in preparation. 
12. J. Williamson, On the equivalence of two singular matrix pencils, Proc. Edin. Math. Soc., Series 2, Volume 4, (1934/36).

Received July 25, 1980.

The UNIVERsity

LIVERPOOL

L69 3BX ENGLAND 



\section{PACIFIC JOURNAL OF MATHEMATICS}

\section{EDITORS}

DONALD BABBITT (Managing Editor)

University of California

Los Angeles, CA 90024

Hugo RossI

University of Utah

Salt Lake City, UT 84112

C. C. MOORE and ARThur Agus

University of California

Berkeley, CA 94720
J. DugundJI

Department of Mathematics

University of Southern California

Los Angeles, CA 90007

R. FINN and J. MILGRAM

Stanford University

Stanford, CA 94305

\section{ASSOCIATE EDITORS}
R. ARENS
E. F. BeCKENBACH
B. H. NeumanN
F. WOLF
K. YoSHIDA

\section{SUPPORTING INSTITUTIONS}

UNIVERSITY OF ARIZONA

UNIVERSITY OF BRITISH COLUMBIA

CALIFORNIA INSTITUTE OF TECHNOLOGY

UNIVERSITY OF CALIFORNIA

MONTANA STATE UNIVERSITY

UNIVERSITY OF NEVADA, RENO

NEW MEXICO STATE UNIVERSITY

OREGON STATE UNIVERSITY

\author{
UNIVERSITY OF OREGON \\ UNIVERSITY OF SOUTHERN CALIFORNIA \\ STANFORD UNIVERSITY \\ UNIVERSITY OF AAWAII \\ UNIVERSITY OF TOKYO \\ UNIVERSITY OF UTAH \\ WASHINGTON STATE UNIVERSITY \\ UNIVERSITY OF WASHINGTON
}

The Supporting Institutions listed above contribute to the cost of publication of this Journal, but they are not owners or publishers and have no responsibility for its content or policies,

Mathematical parers intended for publication in the Pacific Journal of Mathematics should be in typed form or offset-reproduced, (not dittoed), double spaced with large margins. Please do not use built up fractions in the text of the manuscript. However, you may use them in the displayed equations. Underline Greek letters in red, German in green, and script in blue. The first paragraph or two must be capable of being used separately as a synopsis of the entire paper. Please propose a heading for the odd unmbered pages of less than 35 characters. Manuscripts, in triplicate, may be sent to any one of the editors. Please classify according to the scheme of Math. Reviews, Index to Vol. 39. Supply name and address of author to whom proofs should be sent. All other communications should be addressed to the managing editor, or Elaine Barth, University of California, Los Angeles, California, 90024.

50 reprints to each author are provided free for each article, only if page charges have been substantially paid. Additional copies may be obtained at cost in multiples of 50 .

The Pacific Journal of Mathematics is issued monthly as of January 1966, Regular subscription rate: $\$ 114.00$ a year (6 Vol., 12 issues). Special rate: $\$ 57.00$ a year to individual members of supporting institution.

Subscriptions, orders for numbers issued in the last three calendar years, and changes of address shoud be sent to Pacific Journal of Mathematics, P.O. Box 969, Carmel Valley, CA 93924, U.S.A. Old back numbers obtainable from Kraus Periodicals Co., Route 100, Millwood, NY 10546.

\section{PUBLISHED BY PACIFIC JOURNAL OF MATHEMATICS, A NON-PROFIT CORPORATION}

Printed at Kokusai Bunken Insatsusha (International Academic Printing Co., Ltd.). 8-8, 3-chome, Takadanobaba, Shinjuku-ku, Tokyo 160, Japan. 


\section{Pacific Journal of Mathematics}

\section{Vol. 102, No. 2 \\ February, 1982}

Richard A. Boyce, Irreducible representations of finite groups of Lie type through block theory and special conjugacy classes ...............253

Robert Jay Daverman and Dennis J. Garity, Intrinsically

$(n-2)$-dimensional cellular decompositions of $E^{n} \ldots \ldots \ldots \ldots 275$

Juan Ferrera, Spaces of weakly continuous functions ................285

William George Frederick, $\mu$-theta functions ................... 293

Christopher George Gibson and T. D. Ward, On stratifying pairs of linear

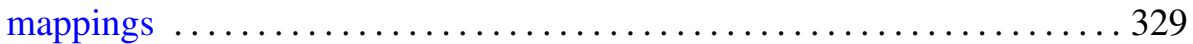

Stanley Joseph Gurak, Minimal polynomials for Gauss circulants and cyclotomic units ........................................ 347

Joachim Georg Hartung, On two-stage minimax problems ............. 355

Robert P. Kaufman, Hausdorff measure, BMO, and analytic functions . . . . 369

Neal I. Koblitz, $p$-adic analog of Heine's hypergeometric $q$-series . . . . . . . 373

Kurt Kreith, Picone-type theorems for hyperbolic partial differential

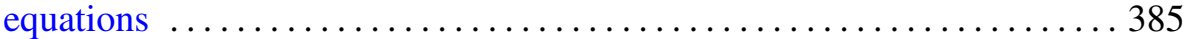

Nicholas J. Kuhn, The geometry of the James-Hopf maps ............. 397

Donald Michael Redmond, Explicit formulae for a class of Dirichlet

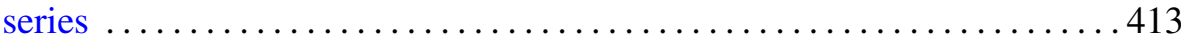

J. R. Respess and Elliott Ward Cheney, Jr., Best approximation problems in tensor-product spaces . .............................. 437

Allen Ross Schweinsberg, The operator equation $A X-X B=C$ with normal $A$ and $B$

Hans-Willi Siegberg and Guentcho Svetoslavov Skordev, Fixed point index and chain approximations

Kondagunta Sundaresan, Geometry and nonlinear analysis in Banach spaces 